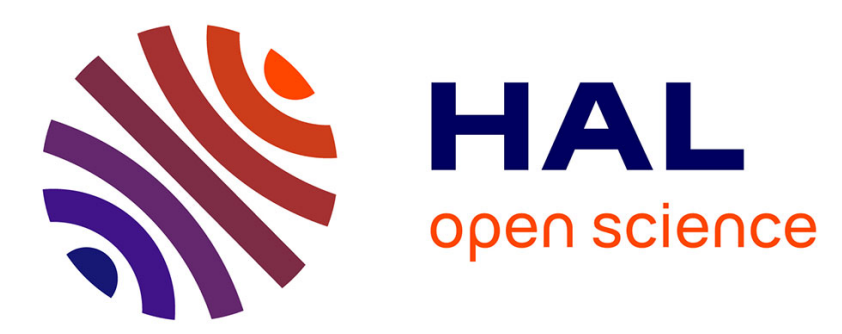

\title{
A computational procedure for predicting the long term residual settlement of a platform induced by repeated traffic loading
}

\author{
M. Abdelkrim, Guy Bonnet, Patrick de Buhan
}

\section{- To cite this version:}

M. Abdelkrim, Guy Bonnet, Patrick de Buhan. A computational procedure for predicting the long term residual settlement of a platform induced by repeated traffic loading. Computers and Geotechnics, 2003, 30 (6), pp.463-476. 10.1016/S0266-352X(03)00010-7 . hal-00691115

\section{HAL Id: hal-00691115 https://hal.science/hal-00691115}

Submitted on 16 Jan 2016

HAL is a multi-disciplinary open access archive for the deposit and dissemination of scientific research documents, whether they are published or not. The documents may come from teaching and research institutions in France or abroad, or from public or private research centers.
L'archive ouverte pluridisciplinaire HAL, est destinée au dépôt et à la diffusion de documents scientifiques de niveau recherche, publiés ou non, émanant des établissements d'enseignement et de recherche français ou étrangers, des laboratoires publics ou privés. 


\title{
A computational procedure for predicting the long term residual settlement of a platform induced by repeated traffic loading
}

\author{
Malek Abdelkrim, Guy Bonnet, Patrick de Buhan* \\ Laboratoire de Matériaux et Structures pour le Génie Civil (LCPC/ENPC/CNRS UMRI13), 6 et 8 av. B. Pascal, Cité Descartes, \\ 77455 Marne-la-Vallée, cedex 2, France
}

\begin{abstract}
A general structural analysis approach is developed in the present paper, allowing the evaluation of the residual settlement of a platform induced by repeated traffic loading. It notably relies upon the formulation of a cyclic constitutive law, which describes the progressive accumulation of irreversible (permanent) deformations locally exhibited by the different underlying granular materials when subjected to long term stress cycling generated by the traffic loading. This constitutive law is incorporated into a step-by-step numerical scheme where two kinds of elastic calculations are implemented: the first one concerns the determination of the so called reference stress cycles, while the second one is aimed at calculating the residual displacement and stress fields of the platform derived from the integration of the permanent non elastic deformations. The whole procedure is illustrated on the simplified model of a moving strip-load acting upon a homogeneous half-space, adopting a cyclic constitutive law formulated for a particular unbound granular material used in road pavements.

Keywords: Traffic loading; Residual settlement; Cyclic loading; Permanent deformations; Unbound granular materials
\end{abstract}

\section{Introduction}

Rational design and maintenance procedures for transportation infrastructures such as road pavements or railway track platforms require the elaboration of predictive methods aimed at assessing the long term performance of this particular kind of geotechnical structures. One of the major specific concern is to be able to determine the residual profile of the infrastructure (pavement rutting, track geometry defects) induced by the application of repeated traffic loading.

In order to get a better insight into the rather complex phenomena involved in this kind of structures, many experimental devices have for instance been developed for testing representative sections of road pavements subject to repeated wheel loading. More recently, an extensive experimental programme has been undertaken on reduced scale models reproducing a segment of railway track subject to cyclic loading [1,2]. Although such experimental approaches are undoubtedly useful for

\footnotetext{
* Corresponding author.

E-mail address: debuhan@1msgc.enpc.fr (P. de Buhan).
}

obtaining a preliminary global understanding of the problem, at least from a qualitative point of view, even making it possible to derive empirical relationships, they remain rather unsuitable for providing genuinely predictive design methods for a wide range of parameters.

The development of calculation methods aimed at predicting the behaviour of geotechnical structures subject to repeated loading requires the formulation of a cyclic constitutive law for the constituent soils. Such a law may be expressed within the framework of (visco)plasticity, involving the usual concepts of yield condition, hardening and flow rules. But the numerical implementation of such a model through a classical step-by-step procedure proves unrealistic beyond a few load cycles, notably because the increment of permanent strain per cycle is rapidly becoming very small, and thus fall below the numerical accuracy of the computational procedure.

Within the general framework proposed by Ponter [3], alternative simplified approaches have been developed based on a "homogenization technique", taking advantage of the fact that the period of repeated load is small when compared to the characteristic creeping time of 
the structure [4,5]. Although the idea of double time scale analysis conveyed in such method will be considered in the following contribution, it should be mentioned that its range of application is restricted to the particular situation of viscoplastic structures undergoing permanent deformations even under constant applied load. This is obviously not the case of traffic platforms where the main source of long term permanent deformations is to be found in the fact that the load is applied repeatedly, that is in a cyclic way, involving a very large number of cycles (up to several millions).

The method developed in the present paper for addressing such a question is based on a "structural analysis" approach, according to which the global response of a traffic platform, and notably its residual surface settlement, may be derived as the solution of a properly defined boundary value problem, provided that the local behaviour of the constituent materials has been previously characterized in terms of stress-strain relationships. More precisely, considering the particular kind of loading involved in the analysis, it clearly appears that such constitutive relationships will refer to the long term cyclic behaviour of the different constituent materials, which exhibit irreversible permanent deformations under cyclic solicitations.

The essential parameters governing this cyclic behaviour may be identified experimentally, on the basis of repeated triaxial loading tests performed on homogeneous samples of material. Constitutive relationships have been proposed for instance by Monosmith et al. [6] or Li and Selig [7], in order to relate the cumulative permanent strain of cohesive subsoils subject to repeated cyclic loading, to the number of load applications as well as the characteristics of the stress cycle (amplitude, proximity to the failure line, etc.). Simplified design procedures, relying on such relationships, have been proposed for road pavements [8] or railway track foundations [9]. Similar constitutive laws have been developed for railroad ballast materials [10,11] or unbound granular materials in the context of road pavement design [12-14]. The latter experiments have required the development of a large triaxial test apparatus, due to the grain size of the granular materials, able to carry out cyclic loading tests up to several tens of thousands cycles.

In contrast with the quite significant amount of experimental data already available for describing the cumulative permanent deformations of granular materials subject to cyclic loading, the calculation methods derived from the incorporation of such data remain somewhat crude. This is for instance due to the fact that, in most cases, the cyclic constitutive law is only referring to the evolution of the axial permanent strain as a function of the number of load cycles, very few indications being given as regards the lateral compo- nent. Yet such an information remains essential for formulating a fully three-dimensional constitutive law and thus devising a rational structural analysis procedure. As a matter of fact, the following contribution is intended to provide the necessary guidelines for defining a general methodology in the framework of continuum mechanics, and thus help improve the present design methods.

\section{Settlement of a platform under traffic loading as a double time scale phenomenon}

Fig. 1 gives a typical representation of the global response of a road pavement or railway structure under traffic loading, namely the evolution of the vertical settlement $\delta$ at a particular point, as a function of time $t=N T$, where $N$ denotes the number of applied wheel loads due to the passage of vehicles, and $T$ is a typical time interval between two such successive loads. It should be emphasized that there is no need to further specify the latter quantity, since dynamic as well as viscoelastic effects will not be considered in the subsequent analysis, so that the only relevant loading parameter is the number $N$ of load cycles and not the physical time $t$ during which they are applied. According to this figure, the observed total deflection may be additively decomposed as:

$\delta(t)=\delta^{e l}(t)+\delta^{r}(t)$

where the first term denotes the rapidly fluctuating elastic deflection which vanishes as the load is removed (that is as the vehicle is moving far away from the point where the settlement is measured), while the second term represents the residual settlement, which slowly increases with time. Furthermore, it clearly appears from such a figure, that the increment of residual settlement over one period of time (say $t \in[N T,(N+1) T]$ ), is considerably smaller than the amplitude of the elastic deflection over the same period (Fig. 1):

$$
\begin{aligned}
& \delta^{r}((N+1) T)-\delta^{r}(N T)<< \\
& \operatorname{Max}\left\{\delta^{e l}(t), \quad t \in[N T,(N+1) T]\right\}
\end{aligned}
$$

so that the settlement could be viewed as a quasi-periodic function of time.

The same kind of observation could be made locally, that is at any point $\underline{x}$ of the structure. Indeed, the stress state at such a point may be written as the superposition of an elastic (recoverable) component due to the applied load and a residual stress:

$\underline{\underline{\sigma}}(\underline{x}, t)=\underline{\underline{\sigma}}^{e l}(\underline{x}, t)+\underline{\underline{\sigma}}^{r}(\underline{x}, t)$

Let us then consider the path followed by such a stress state in the space of stresses, as sketched in Fig. 2. 

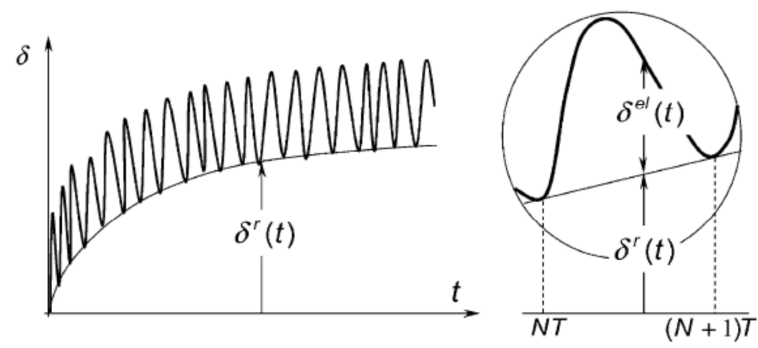

Fig. 1. Evolution of the global settlement of a platform subject to repeated traffic loading.

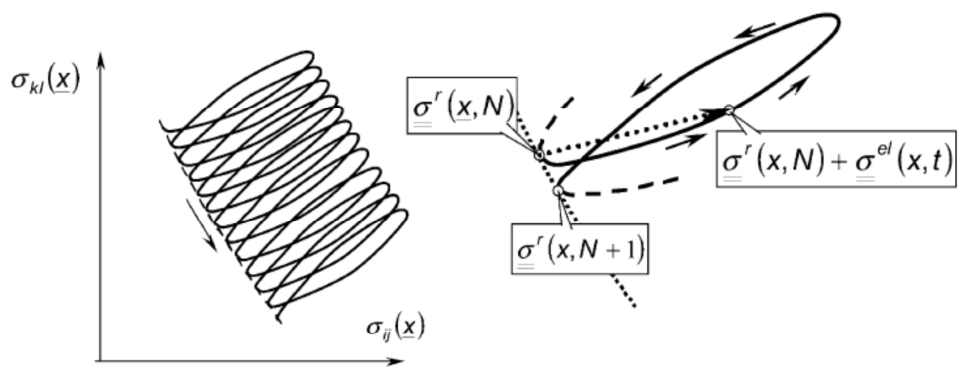

Fig. 2. Stress path at any point of the structure.

It may be drawn as a chain of loops which corresponds to the elastic load cycles slightly drifting as the number $N$ of applied load cycles increases. The distance between two such successive loop-cycles appears to be quite small when compared with the loop size itself. In other terms, the increment of the residual stress per load-cycle is negligible in comparison with the amplitude of the elastic cycle:

$$
\begin{aligned}
\left\|\underline{\underline{\sigma}}^{r}(\underline{x},(N+1) T)-\underline{\underline{\sigma}}^{r}(\underline{x}, N T)\right\|<< \\
\quad \operatorname{Max}\left\{\left\|\underline{\underline{\sigma}}^{e l}(t)\right\|, t \in[N T,(N+1) T\}\right\}
\end{aligned}
$$

Nevertheless, it should be kept in mind that, in spite of (2), the amount of residual settlement accumulated at the end of $N$ load-cycles (with $N$ up to several millions) may become of the same order as or even greater than the elastic deflection. The same remark applies to the variation of residual stress as compared to the elastic stress amplitude.

The two-scale time evolution of the settlement of a platform observed under repeated traffic loading due to the passage of a moving load is quite reminiscent of the liquefaction phenomenon of soil masses subject to seismic or wave loading $[15,16]$. Indeed, as already pointed out, this evolution may be described by a quasi-periodic function of time, in much the same way as, in a quite different context, the curves representing the evolution of excess pore pressure progressively building up in a saturated granular soil sample under deviatoric cyclic loading, and ultimately leading up to liquefaction when the effective stress drops to zero. It has been clearly shown that the physical explanation of such a progressive accumulation of excess pore pressure is to be found in the soil's volume decrease (contraction) generated by cyclic loading [15].

The fundamental assumption upon which the analysis and related design method developed in this paper are based, is that the long term behaviour of a traffic platform observed at both the global (progressive accumulation of residual settlement) and local levels (drift of residual stresses), is to be attributed to the cyclic behaviour of the different granular materials which constitute the platform: ballast, gravel, cohesionless soils, etc. More precisely, those materials undergo irreversible (permanent) strains when subjected to repeated loadings, as shown for instance by Alva-Hurtado [11] and Hornych et al. [12], who performed repeated triaxial tests on ballast material and pavement unbounded granular materials, respectively.

Despite the obvious similarity between the liquefaction and platform settlement prediction problems as regards the underlying mechanical behaviour of the constituent materials, there remains some important differences which are listed below.

- The granular materials which constitute the platform are generally not liable to liquefaction, even in the case of saturated subgrade layers, since the excess pore pressures are almost immediately dissipated. 
- While in the liquefaction problem only the irreversible volume change is to be taken into account in the analysis, all components of the materials permanent strains should be considered for predicting the platform residual settlement, as it will be seen later on.

- The number of load cycles associated with the traffic loading is far more important than for soil liquefaction: several millions cycles against a few hundreds.

- Finally, it seems likely that the physical mechanisms which prevail at the microscopic scale for explaining the observed permanen deformations are different in both cases. Densification by rearrangement of an initially loose assembly of grains is the governing mechanism in the liquefaction problem, whereas if such a mechanism may explain the observed residual settlement of a traffic platform in an initial phase (say a for a few thousands cycles), other phenomena such as the attrition of grains or particle crushing seem to be the main source of long term permanent deformations.

\section{A general framework for calculating the residual platform settlement}

The starting point for setting up a general design method for predicting the residual settlement of a platform under repeated traffic loading lies in the formulation of a constitutive law for the different materials subject to cyclic loading. Such a cyclic constitutive law may be expressed in the form of a local relationship between the rate of accumulation of permanent strain at the $N^{\text {th }}$ load cycle and the characteristics of the cyclic loading path, namely:

$\frac{\mathrm{d} \varepsilon^{p}}{=}(\underline{\mathrm{d} N}, N)=F[N ; \quad(\oint \underline{\underline{\sigma}})(\underline{x}, N)]$

In the above equation:

- $\underline{\varepsilon}^{p}(\underline{x}, N)$ is the permanent strain experienced by the material located at point $\underline{x}$ and at the end of $N$ applied cycles;

- $(\oint \underline{\underline{\sigma}})(\underline{x}, N)$ denotes the stress cycle to which the material located at the same point $\underline{x}$ is submitted at the same time $t=N T$.

The subsequent developments are based on the two following assumptions

- The short term reversible behaviour of the different materials is described by a linear elastic constitutive law.
- The elastic stiffness parameters (that is the Young modulus and Poisson ratio in the case of isotropy) remain unaffected by the cyclic loading. This means that no damage or stiffening is taken into account.

While the second assumption appears to be reasonably well verified once a few load cycles have been applied, the first one seems more questionable, since experimental evidence of a strong non linearity has been given for some granular materials.

As an important consequence of theses hypotheses, it appears from the superposition principle that the current stress cycle actually applied to the material is obtained in the stress space from translating the so called reference stress cycle by the residual stress (Fig. 3).

$(\oint \underline{\underline{\sigma}})(\underline{x}, N)=\underline{\underline{\sigma}}^{r}(\underline{x}, N)+(\oint \underline{\underline{\sigma}})^{\mathrm{ref}}(\underline{x})$

It is to be noted that the reference stress cycle is a function of point $\underline{x}$ only. It is simply obtained as the sequence of stress states generated at that point as the load is moving on the platform surface, which may be derived from the solution of a simple elastic boundary value problem.

It appears from this preliminary analysis that the proposed method for determining the residual state of the structure and notably the cumulated settlement as a function of the traffic loading, should therefore combine three complementary elements:

(a) The formulation of a cyclic constitutive law in the form of Eq. (5), which has to be derived from laboratory tests conducted for each type of material and for various cyclic loading conditions.

(b) A computational tool for determining the reference stress cycle at any point of the structure as a function of the traffic loading characteristics.

(c) A numerical procedure for calculating the residual state of the structure, and more particularly the residual settlement, from the knowledge of the accumulated permanent strain field.

Procedure (c) amounts to solving an elastic boundary value problem relating to the same structure, in the absence of the load traffic, but with the permanent strain field acting as a prescribed non-elastic strain field, in exactly the same way as for thermal or plastic strains. The existence, and subsequent evolution, of the residual stress field is thus clearly connected with the geometrical non-compatibility of this strain field.

Since the cyclic constitutive formulation is given in the incremental form (5), the implementation of such a method requires the discretization of the total number of applied load cycles into a finite number steps: 


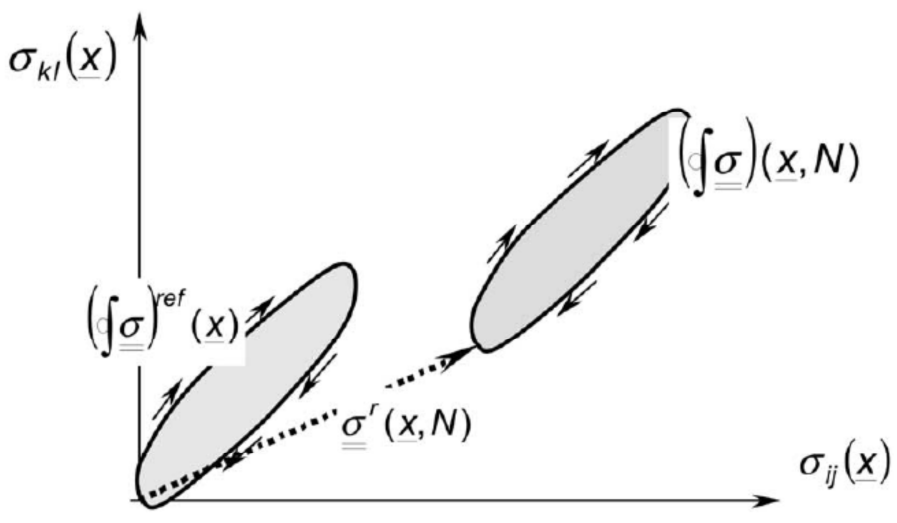

Fig. 3. Reference and current stress cycles at point $\underline{x}$.

$N=k \Delta N, k=0,1, . . K$, where $\Delta N$ may range between a few units and several thousands. The principle of the corresponding numerical algorithm, which is sketched in Fig. 4, is described as follows:

- A preliminary elastic calculation is first carried out which provides once and for all the reference stress cycle at any point of the structure from the moving load characteristics.

- The algorithm itself is getting started from $k=0$, where the residual state of the structure coincides with its given initial state: the permanent strain field and associated residual settlement are conventionally taken equal to zero, while the residual stress field is equal to the initial stress field, which must be in equilibrium with the gravity loads:

$\delta^{r}(k=0)=0, \quad \underline{\varepsilon}^{p}(k=0)=0, \quad \underline{\underline{\sigma}}^{r}(k=0)=\underline{\underline{\sigma}}^{0}$

- Assume now that the permanent strain field along with the residual state are known up to step $\mathrm{n}^{\circ} k$. The local value of the permanent strain field at step $\mathrm{n}^{\circ} k+1$ is simply computed as the sum of its value at the previous step and of the increment given by $\mathrm{Eq}$. (5), where $\mathrm{d} N$ is replaced by $\Delta N$ and $\quad \mathrm{d} \underline{\varepsilon}^{p}(N)$ approximated by $\underline{\underline{\varepsilon}} p(\underline{x}, k+1)-\underline{\varepsilon}^{p}(\underline{x}, k)$, so that:

$\underline{\underline{\varepsilon}} \underline{\underline{\varepsilon}}(\underline{x}, k+1)=\underline{\underline{\varepsilon}} \underline{\varepsilon}^{p}(\underline{x}, k)+\Delta N$

$$
\times F\left[N=k \Delta N ; \underline{\underline{\sigma}}^{r}(\underline{x}, k)+(\oint \underline{\underline{\sigma}})^{\mathrm{ref}}(\underline{x})\right]
$$

- The residual stress field at step $\mathrm{n}^{\circ} k+1$ is then determined from solving the elastic boundary value problem with no traffic loading but incorporating $\varepsilon^{p}(k+1)$ as a prescribed non elastic strain field.

- The computing procedure is continued until $k$ reaches its maximum value $K$.

\section{An illustrative application: platform under moving strip-load}

In order to demonstrate how the proposed method and related numerical algorithm can be implemented,

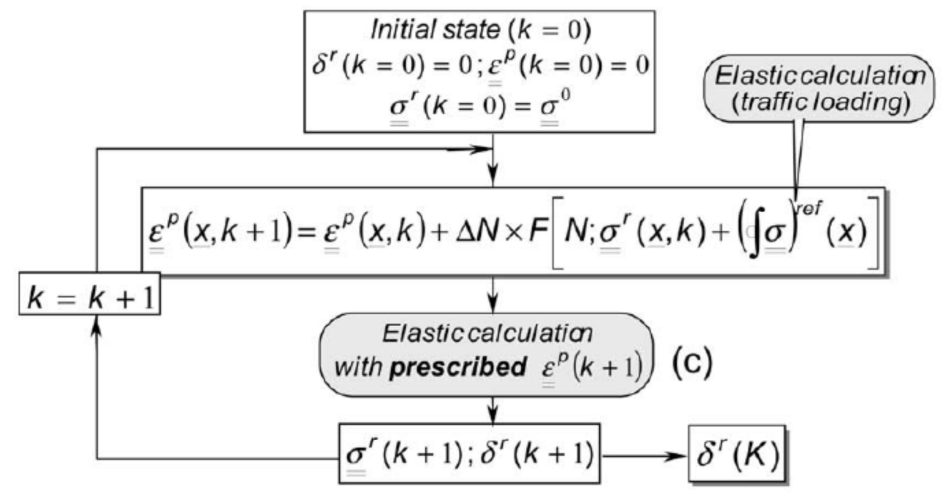

(b)

Fig. 4. Principle of the numerical algorithm. 


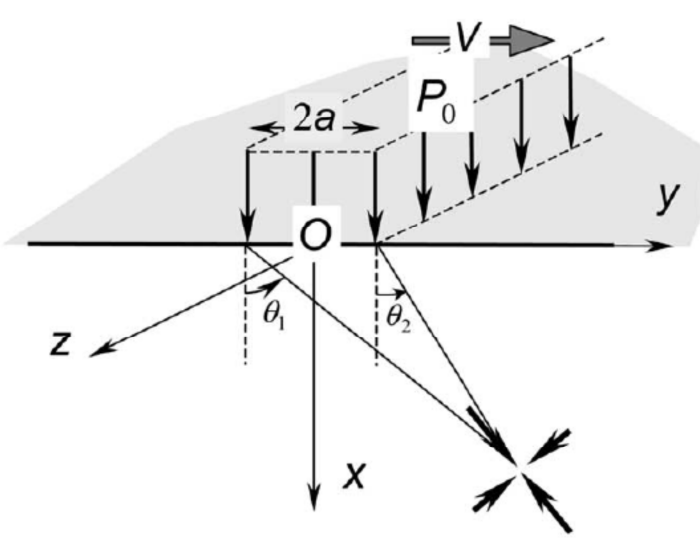

Fig. 5. Simplified model of a traffic platform subject to traffic loading.

the following simplified schematization of a platform under traffic loading will be adopted. The load which represents the action of the passing vehicle is acting upon a homogeneous half-space in the form of a constant downwards pressure $P_{0}$ applied over a strip of width $2 a$ and infinite length, as shown in Fig. 5, and moving with a velocity $V$ along the $O y$-axis, the cartesian $O x y$-frame being attached to the moving load.

It is assumed that the constituent material behaves as an homogeneous isotropic linear elastic medium (no creep behaviour is taken into account), and that all dynamic effects are neglected. It then appears that for an initially stress-free unloaded structure, the elastic state of stress created at any point $(x, y, z)$ by the application of such a strip-load, is classically obtained from integrating the elementary Boussinesq's solution over the strip's width. The different stress components are [17]:

$$
\left\{\begin{array}{c}
\sigma_{x x}=\left(P_{0} / \pi\right)\left[\theta_{1}-\theta_{2}+1 / 2\left(\sin 2 \theta_{1}-\sin 2 \theta_{2}\right)\right] \\
\sigma_{x y}=\left(P_{0} / 2 \pi\right)\left[\cos 2 \theta_{2}-\cos 2 \theta_{1}\right] \\
\sigma_{y y}=\left(P_{0} / \pi\right)\left[\theta_{1}-\theta_{2}-1 / 2\left(\sin 2 \theta_{1}-\sin 2 \theta_{2}\right)\right] \\
\sigma_{z z}=2 \nu\left(P_{0} / \pi\right)\left[\theta_{1}-\theta_{2}\right] \text { other } \sigma_{i j}=0
\end{array}\right.
$$

where angles $\left(\theta_{1}, \theta_{2}\right)$ are defined as (Fig. 5):

$\theta_{1}=\tan ^{-1}\left(\frac{y+a}{x}\right) \quad \theta_{2}=\tan ^{-1}\left(\frac{y-a}{x}\right)$

and $v$ is the Poisson's ratio. The corresponding expressions of the two first stress invariants defined as:

$p=-\operatorname{tr} \underline{\underline{\sigma}} / 3$ and $q=\left(3 / 2 \operatorname{tr}_{\underline{\underline{s}}}^{2}\right)^{1 / 2}$

where $\underline{\underline{s}}=\underline{\underline{\sigma}}-p \underline{\underline{I}}$ is the deviatoric stress, are:

$\left\{\begin{array}{l}p=\frac{2 P_{0}}{3 \pi}(1+v)\left[\theta_{1}-\theta_{2}\right] \\ q=\frac{P_{0}}{\pi}\left[(1-2 \nu)^{2}\left(\theta_{1}-\theta_{2}\right)^{2}+3 \sin ^{2}\left(\theta_{1}-\theta_{2}\right)\right]^{1 / 2}\end{array}\right.$ so that the contour surfaces of $(p, q)$ are formed by the loci of points such that $\theta_{1}-\theta_{2}$ remains constant, which is the family of circular cylindrical surfaces passing through the lines of equations $x=0, y= \pm a$, and whose axes are located in the symmetry plane $O x z$. Considering then any fixed point at a depth $x$ below the surface, the reference stress cycle to which such a point is subjected as the load is passing by, corresponds to the stress distribution given by (9) and (10) along the horizontal line passing through this point and parallel to the $O y$-axis

$$
(\oint \underline{\underline{\sigma}})^{\mathrm{ref}}(x)=\{\underline{\underline{\sigma}}(x, y), y:+\infty \text { to }-\infty\}
$$

The associated stress path in the $(p, q)$-plane is therefore determined from Eqs. (12) where the angular parameter $\theta_{1}-\theta_{2}$ increases from 0 to the maximum value $2 \tan ^{-1}(a / x)$ as $y$ decreases from $+\infty$ to 0 (approaching load), then goes back to 0 as $y$ tends towards $-\infty$, that is as the load is moving away. This stress path is located on the curve of equation

$$
\begin{aligned}
& q / P_{0}=\frac{1}{\pi}\left[(1-2 v)^{2}\left(\frac{3 \pi p}{2(1+v) P_{0}}\right)^{2}\right. \\
& \left.+3 \sin ^{2}\left(\frac{3 \pi p}{2(1+v) P_{0}}\right)\right]^{1 / 2}
\end{aligned}
$$

obtained from eliminating $\theta_{1}-\theta_{2}$ between the parametric Eqs. (12). It connects the origin to the extreme point whose normalized co-ordinates are

$$
\left\{\begin{aligned}
p_{\text {ref }} / P_{0} & =\frac{4}{3 \pi}(1+v) \tan ^{-1}(a / x) \\
q_{\text {ref }} / P_{0} & =\frac{1}{\pi}\left[(1-2 v)^{2}\left(2 \tan ^{-1}(a / x)\right)^{2}\right. \\
+ & \left.3 \sin ^{2}\left(2 \tan ^{-1}(a / x)\right)\right]^{1 / 2}
\end{aligned}\right.
$$



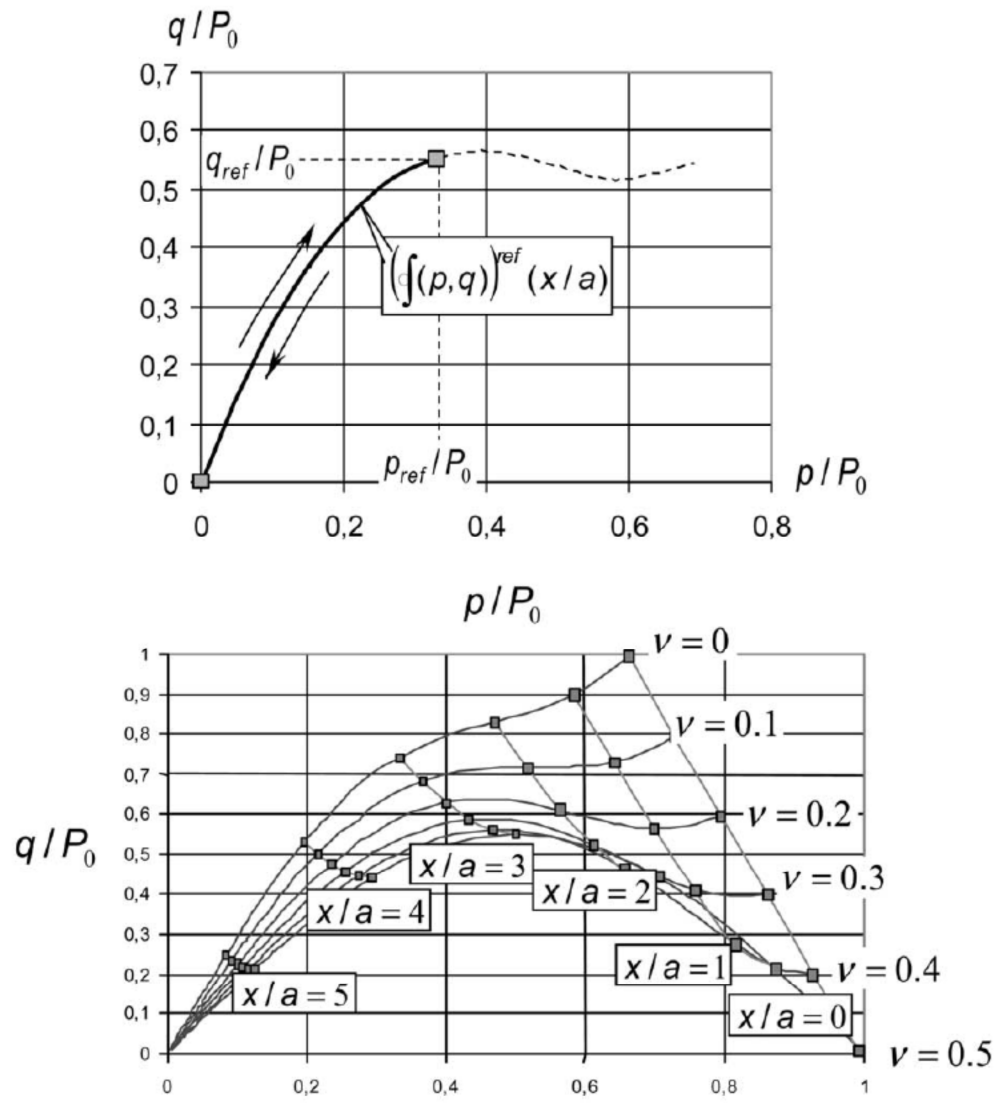

Fig. 6. Normalised reference stress cycles as a function of $x / a$ and $v$.

Those reference stress cycles are represented in Fig. 6. They depend on the Poisson's ratio $v$ and the relative depth $x / a$ only. For a given value of $v$, they are located on the same curve [Eq. (14)], their amplitude being a decreasing function of the relative depth.

\section{A cyclic constitutive law for modelling permanent} deformations

The general procedure outlined in section 3 requires the specification of a constitutive law aimed at relating the production of permanent deformations exhibited by the material under repeated loading, to the number of applied cycles along with some relevant characteristics of these cycles such as their position or amplitude in the stress space. The cyclic constitutive law we shall adopt in the present analysis, is directly inspired from that formulated by Gidel et al. [14] on the basis of repeated loading triaxial tests performed on unbound granular materials (UGM), such as Poulmarch and Soreze gravels. Those triaxial tests were designed in such a way that both the axial stress and confining pressure could be varied, thus allowing to explore the material cyclic behaviour along different radial stress paths such as that sketched in Fig. 7 in the $(p, q)$-space.

The cyclic constitutive law proposed by Gidel et al. [14] for modelling the permanent deformation

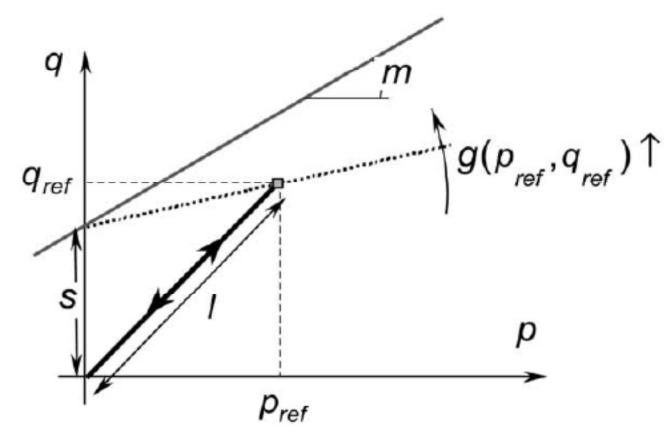

Fig. 7. Cyclic constitutive law proposed by Gidel et al. [14] for unbound granular materials subject to radial load cycles. 
behaviour of the Poulmarch gravel may be expressed as follows:

$$
\begin{aligned}
\varepsilon_{1}^{p} & =\varepsilon_{0}^{p}\left(1-N^{-B}\right) f\left(p_{\text {ref }}, q_{\text {ref }}\right) . g\left(p_{\text {ref }}, q_{\text {ref }}\right), \\
N & \geqslant 1
\end{aligned}
$$

where $\varepsilon_{1}^{p}$ is the accumulated axial permanent strain due to the application of $N$ stress cycles characterised by the stress peak values. Functions $f$ and $g$ are defined as follows

$$
\left\{\begin{array}{l}
f\left(p_{\text {ref }}, q_{\text {ref }}\right)=(l / 100)^{n} \text { with } l=\sqrt{p_{\text {ref }}^{2}+q_{\text {ref }}^{2}} \\
g\left(p_{\text {ref }}, q_{\text {ref }}\right)=\frac{p_{\text {ref }}}{s+m p_{\text {ref }}-q_{\text {ref }}}
\end{array}\right.
$$

where $\varepsilon_{0}^{p}, B, n, s$ and $m$ are material parameters, all stress quantities involved in the different formulas being expressed in $\mathrm{kPa}$. Function $f$ represents the influence of the stress cycle amplitude $l$ on the amount of axial permanent strain, while function $g$ quantifies the proximity of the peak stress point $\left(p_{\text {ref }}, q_{\text {ref }}\right)$ with respect to a kind of "ultimate line" whose equation is $q=s+m p$ (Fig. 7). More precisely, the contour lines of $g$ are straight lines intersecting the $q$-axis at the same point as the "ultimate line", so that the value of function $g$ tends to infinity as the peak stress point approaches this ultimate line.

Although the previous cyclic law has been established for radial stress cycles only, the following extension to curve-shaped stress cycles generated by the moving strip-load problem is now proposed. Fig. 8 shows a typical current stress cycle obtained from translating the reference stress cycle determined in Section 4, which starts from the origin, by the residual state of stress $\left(p^{r}, q^{r}\right)$. The cyclic constitutive law (15) may be generalised as:

$\varepsilon_{1}^{p}(N)=\varepsilon_{0}^{p}\left(1-N^{-B}\right) f\left(p_{\text {ref }}, q_{\text {ref }}\right) \cdot g\left(p^{r}+p_{\text {ref }}, q^{r}+q_{\text {ref }}\right)$

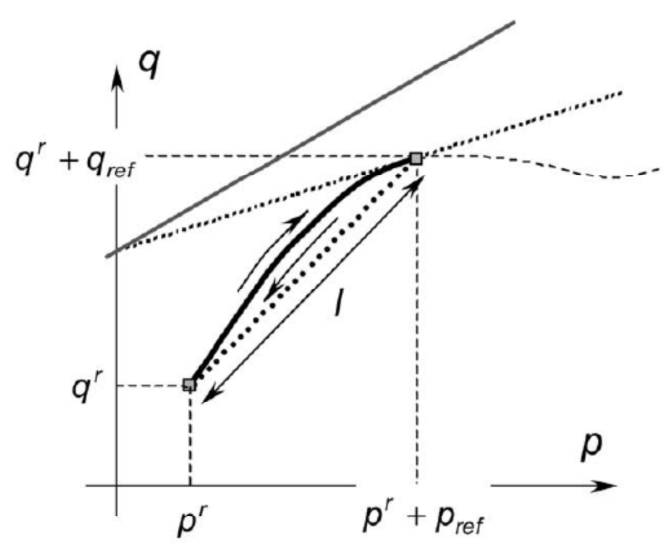

Fig. 8. Extension of the cyclic constitutive law to current stress cycles generated in the moving load problem.
This formula is valid for $N$ load cycles applied between $\left(p^{r}, q^{r}\right)$ and $\left(p^{r}+p_{\text {ref }}, q^{r}+q_{\mathrm{ref}}\right)$ considered as fixed values in the stress space. Now, due to the continuous evolution of the permanent strain field, the residual stresses $\left(p^{r}, q^{r}\right)$ generated by the geometric incompatibility of permanent strains (see following section), are progressively varying as $N$ increases. Therefore, the above cyclic constitutive law should be formulated in an incremental form, as follows. The increment of permanent axial strain due to the application of $\Delta N$ stress cycles between $\left(p^{r}, q^{r}\right)(N)$ and $\left(p^{r}+p_{\text {ref }}, q^{r}+q_{\text {ref }}\right)(N)$ may be written as:

$$
\begin{aligned}
\Delta \varepsilon_{1}^{p}(N) & =\varepsilon_{1}^{p}(N+\Delta N)-\varepsilon_{1}^{p}(N) \\
& =\varepsilon_{0}^{p}\left(N^{-B}-(N+\Delta N)^{-B}\right) f\left(p_{\mathrm{ref}}, q_{\mathrm{ref}}\right) \cdot g\left(p^{r}(N)\right. \\
& \left.+p_{\mathrm{ref}}, q^{r}(N)+q_{\mathrm{ref}}\right)
\end{aligned}
$$

In order to obtain a complete formulation of the cyclic constitutive law which could be incorporated in the general calculation procedure described in Section 3, it is necessary to specify the evolution of the other principal strain components $\varepsilon_{2}^{p}=\varepsilon_{3}^{p}$. Actually, referring more particularly to triaxial tests, it appears that few experimental data are available concerning the lateral components of the permanent deformations experienced by samples of granular materials subject to repeated load cycles. However, preliminary investigations seem to indicate that those lateral components are varying proportionally to the axial one, but with the opposite sign. This means for instance that a sample of material, subject to a compressive cyclic test, expands laterally $\varepsilon_{2}^{p}=\varepsilon_{3}^{p} \geqslant 0$, whereas it undergoes an axial contraction $\varepsilon_{1}^{p} \leqslant 0$. Therefore, as a first tentative to derive a complete three dimensional formulation for a cyclic constitutive, we shall assume that the following relationship between the axial and lateral permanent strain components applies

$\varepsilon_{2}^{p}=\varepsilon_{3}^{p}=-v^{*} \varepsilon_{1}^{p}$

where $v^{*}$ is a non-dimensional parameter, which could be interpreted as a kind of Poisson's ratio relating to the permanent (instead of elastic) deformations.

It could be interesting to connect this parameter to the concept of dilatancy-contractancy classically introduced for describing the plastic volume change of soils. Let

$\varepsilon_{v}^{p}=\operatorname{tr}_{\underline{\underline{\varepsilon}}}^{p}=\varepsilon_{1}^{p}+2 \varepsilon_{2}^{p}=\left(1-2 v^{*}\right) \varepsilon_{1}^{p}$

denote the permanent volumetric strain. Since $\varepsilon_{1}^{p} \leqslant 0$, it clearly appears that the material is contractant when $v^{*}<1 / 2$, dilatant for $v^{*}>1 / 2$, while exhibiting no volume change when $v^{*}=1 / 2$. Even though some experimental results [14] tend to show that $v^{*}$ is an increasing function of the stress ratio $q^{\text {ref }} / p^{\text {ref }}$, meaning 
that the material is all the more dilatant (or less contractant) as the slope of the stress path in the $(p, q)$ plane is important, it will be assumed here, as a first approximation, that it is a material constant.

\section{Determination of the residual displacement and stress fields}

Let $\underline{\varepsilon}^{p}(\underline{x} ; N)$ be the permanent strain tensor accumulated at point $\underline{x}$ of the traffic platform at the end of $N$ load cycles. The determination of the residual displacement and stress fields, and notably of the residual surface settlement consists in solving an elastic boundary value problem without any traffic load, but with $\varepsilon^{p}(N)$ acting as a prescribed non-elastic strain field. precisely, denoting by $\underline{\xi}^{r}(N)$ and $\underline{\underline{\sigma}}^{r}(N)$ the residual displacement and stress solutions, the following set of equations should be satisfied.

Elastic constitutive behaviour:

$$
\begin{aligned}
& \underline{\underline{\sigma}}^{r}(\underline{x} ; N)=\underline{\underline{\sigma}}^{r}(\underline{x} ; N=0) \\
& +\lambda \operatorname{tr}\left(\underline{\underline{\varepsilon}}(\underline{x} ; N)-\underline{\underline{\varepsilon}}^{p}(\underline{x} ; N)\right) \underline{\underline{I}} \\
& ++2 \mu\left(\underline{\underline{\varepsilon}}^{r}(\underline{x} ; N)-\underline{\underline{\varepsilon}}^{p}(\underline{x} ; N)\right) \\
& \text { with } \underline{\underline{\varepsilon^{r}}}(\underline{x} ; N)=1 / 2\left(\underline{\underline{\operatorname{grad} \xi^{r}}}+{ }^{T} \underline{\underline{\operatorname{grad}} \xi^{r}}\right)(\underline{x} ; N)
\end{aligned}
$$

where $\underline{\underline{\sigma}}^{r}(N=0)$ is the initial stress field. $\lambda$ and $\mu$ are the Lamé's constants.

Equilibrium equation:

$\operatorname{div} \underline{\underline{\sigma}}^{r}(\underline{x} ; N)+\underline{\gamma}=0$

where $\gamma$ is the specific weight of the material.

Boundary condition: stress-free surface.

$\underline{\underline{\sigma}}^{r}(x=0 ; N) \cdot \underline{e}_{x}=0$

The form of the permanent strain tensor can be specified as follows. Even though, during a load cycle, the principal directions of the stress at any point rotate as the load is moving, it may be deduced from obvious symmetry considerations that the principal directions of the permanent strain generated from such a cycling load remain coincident with the vertical and horizontal axes. Hence necessarily

$$
\begin{aligned}
\underline{\varepsilon}^{p}(\underline{x}, N)= & \varepsilon_{1}^{p}(x, N) \underline{e}_{x} \otimes \underline{e}_{x}+\varepsilon_{2}^{p}(x, N) \\
& \times\left(\underline{e}_{y} \otimes \underline{e}_{y}+\underline{e}_{z} \otimes \underline{e}_{z}\right)
\end{aligned}
$$

where the principal vertical $\varepsilon_{1}^{p}$ and horizontal $\varepsilon_{2}^{p}=\varepsilon_{3}^{p}$ components depend on the depth $x$ only. Their evolution is governed by the cyclic constitutive Eqs. (18) and (19).
The residual displacement field is then searched in the following form:

$\underline{\xi}^{r}=u^{r}(x, N) \underline{e}_{x}$

so that, assuming that the platform is initially stress-free $\left(\sigma^{r}(x, N=0)=0\right)$, Eq. (21) becomes:

$$
\begin{aligned}
& \underline{\underline{\sigma}}^{r}(\underline{x} ; N)=\lambda \operatorname{tr}\left(\underline{\underline{\varepsilon}}(\underline{x} ; N)-\underline{\underline{\varepsilon}}^{p}(\underline{x} ; N)\right) \underline{\underline{I}} \\
& +2 \mu(\underline{\underline{\varepsilon}}(\underline{x} ; N)-\underline{\underline{\varepsilon}} \underline{\underline{\varepsilon}}(\underline{x} ; N)) \\
& \text { with } \quad \underline{\underline{\varepsilon}} \underline{\underline{x}}(\underline{x} ; N)=\frac{\mathrm{d} u^{r}}{\mathrm{~d} x}(x ; N) \underline{e}_{x} \otimes \underline{e}_{x}
\end{aligned}
$$

It thus follows from (24) that the residual stress components are

$\left\{\begin{array}{l}\sigma_{x x}^{r}=\lambda\left(\frac{\mathrm{d} u^{r}}{\mathrm{~d} x}-\varepsilon_{1}^{p}-2 \varepsilon_{2}^{p}\right)+2 \mu\left(\frac{\mathrm{d} u^{r}}{\mathrm{~d} x}-\varepsilon_{1}^{p}\right) \\ \sigma_{y y}^{r}=\sigma_{z z}^{r}=\lambda\left(\frac{\mathrm{d} u^{r}}{\mathrm{~d} x}-\varepsilon_{1}^{p}-2 \varepsilon_{2}^{p}\right)+2 \mu\left(-\varepsilon_{1}^{p}\right)\end{array}\right.$ other $\sigma_{i j}=0$

Substituting these expressions into the equilibrium Eq. (22), where the specific weight is taken equal to zero, yields:

$\operatorname{div} \underline{\underline{\sigma^{r}}}=0 \Rightarrow \frac{\mathrm{d} \sigma_{x x}^{r}}{\mathrm{~d} x}=0 \Rightarrow \sigma_{x x}^{r}=0$

where the boundary condition (23) has been taken into account. Consequently

$\frac{\mathrm{d} u^{r}}{\mathrm{~d} x}=\varepsilon_{1}^{p}(x)+\frac{2 \lambda}{\lambda+2 \mu} \varepsilon_{2}^{p}(x)=\varepsilon_{1}^{p}(x)+\frac{2 v}{1-v} \varepsilon_{2}^{p}(x)$

Since it is reasonable to assume that the permanent strains are negligible beyond a sufficient depth $H>>2 a$, so that the residual settlement can be taken equal to zero at such a depth, Eq. (29) can be integrated into:

$u^{r}(x ; N)=-\int_{\chi=x}^{\chi=H}\left[\varepsilon_{1}^{p}(\chi)+\frac{2 v}{1-v} \varepsilon_{2}^{p}(\chi)\right] \mathrm{d} \chi$

which gives in particular the expression of the residual surface settlement:

$$
\begin{aligned}
\delta^{r}(N) & =u^{r}(x=0 ; N) \\
& =-\int_{x=0}^{x=H}\left[\varepsilon_{1}^{p}(x ; N)+\frac{2 v}{1-v} \varepsilon_{2}^{p}(x ; N)\right] \mathrm{d} x
\end{aligned}
$$


Finally substituting (29) into (27) provides the expressions for the different residual stress components as a function of the sole lateral permanent strain:

$$
\begin{aligned}
\sigma_{y y}^{r}(x ; N) & =\sigma_{z z}^{r}(x ; N)=-\frac{2 \mu(2 \mu+3 \lambda)}{\lambda+2 \mu} \varepsilon_{2}^{p}(x ; N) \\
& =-\frac{E}{1-v} \varepsilon_{2}^{p}(x ; N)
\end{aligned}
$$

other $\sigma_{i j}^{r}=0$

The corresponding residual stress invariants, calculated from (11), are:

$$
\begin{aligned}
p^{r}(x ; N) & =-1 / 3 \operatorname{tr}\left(\underline{\underline{\sigma^{r}}}(x ; N)\right)=\frac{2 E}{3(1-v)} \varepsilon_{2}^{p}(x ; N) \\
q^{r}(x ; N) & =\left[3 / 2 \operatorname{tr}\left(\underline{\underline{s^{r}}}(x ; N)\right)^{2}\right]^{1 / 2}=\frac{E}{(1-v)}\left|\varepsilon_{2}^{p}(x ; N)\right| \\
& =\frac{3}{2}\left|p^{r}(x ; N)\right|
\end{aligned}
$$

\section{Numerical implementation and results}

The incremental numerical algorithm described in general terms in Fig. 4 has been implemented for the simplified model of traffic platform under repeated moving load: Fig. 9. All steps of calculations are carried out in an analytical way for each depth $x$ independently, the resulting residual surface settlement $\delta^{r}(N)$ being calculated from a classical integration procedure of the permanent strain distributions down to a maximum depth $H / a=50$ [Eq. (31)]. The following set of data has been selected for the simulation:

$P_{0}=300 \mathrm{kPa}, E=100 \mathrm{MPa}, v=0.3, v^{*}=0.6$,

$\varepsilon_{0}^{p}=-0.02, \quad B=0.03, \quad n=0.588, \quad m=3.8$,

$s=42.8 \mathrm{kPa}$ where the last five parameters correspond to the values identified on a particular granular material (Poulmarch gravel) by Gidel et al. [14] on the basis of repeated triaxial loading tests.

Fig. 10 displays different curves giving the evolution of the accumulated vertical permanent strain as the number of load cycles is increasing up to one million. It can be seen from this figure that for the selected set of material and loading parameters (34), the permanent strain is negative (vertical contraction), the amplitude of such a contraction being, as could be expected, an increasing function of $N$ and a decreasing function of the relative depth $x / a$. Since the coefficient $v^{*}$ has been chosen equal to 0.6, it follows from Eq. (19) that the horizontal permanent strain is always positive, corresponding to an extension. Eq. (32) shows that such a distribution of horizontal permanent strains produces compressive horizontal residual stresses.

$\sigma_{y y}^{r}(x ; N)=\sigma_{z z}^{r}(x ; N)=\frac{v^{*} E}{1-v} \varepsilon_{1}^{p}(x ; N) \leqslant 0$

Fig. 11(a) represents the corresponding residual stress profiles for increasing values of $N$, while Fig. 11(b) gives for different depth levels the associated residual stress paths in the $(p, q)$-plane, corresponding to the progressive drift of the origin of the current stress cycles, and their position with respect to the "ultimate line" of equation $q=42.8+3.8 p$ which has been plotted in the same figure. It is to be noted that those paths are represented by segments placed along the same straight line of equation $q=3 p / 2$, their amplitude for a given number of load cycles being a decreasing function of depth. As a final global result of such calculations, the non dimensional residual settlement is represented in Fig. 12 as an increasing function of $N$.

Starting from the reference set of data given by (34), a parametric study has been undertaken, in which both

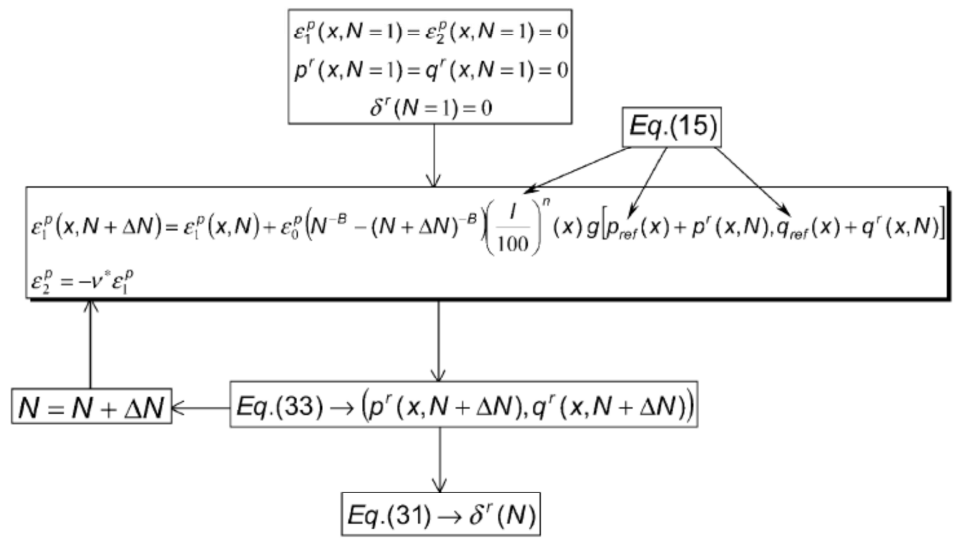

Fig. 9. Step-by-step procedure for calculating the residual settlement of a platform under repeated traffic loading. 


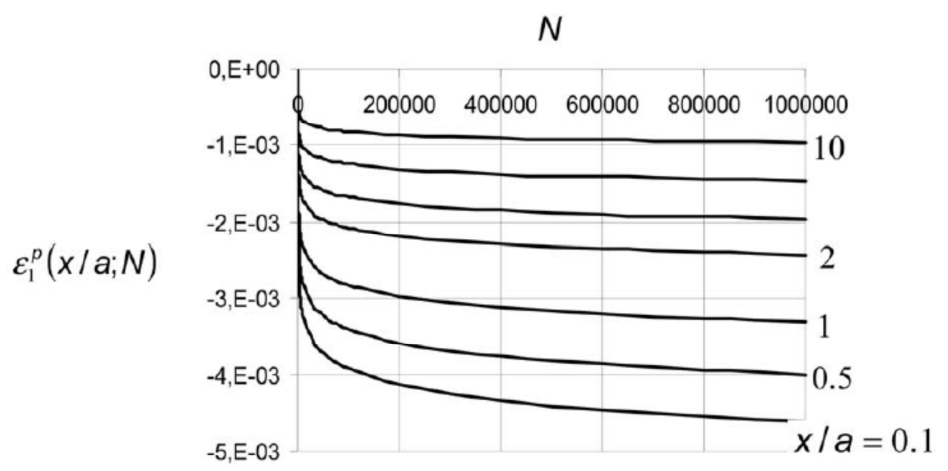

Fig. 10. Evolution of vertical permanent strain as a function of $N$ at different relative depths $x / a$.

(a) $\sigma_{y y}^{r}=\sigma_{z z}^{r}(\mathrm{kPa})$

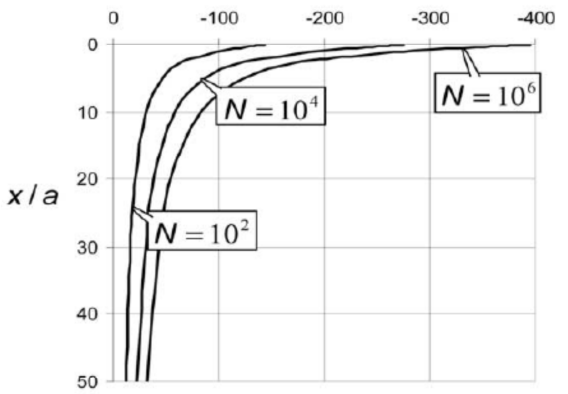

(b)

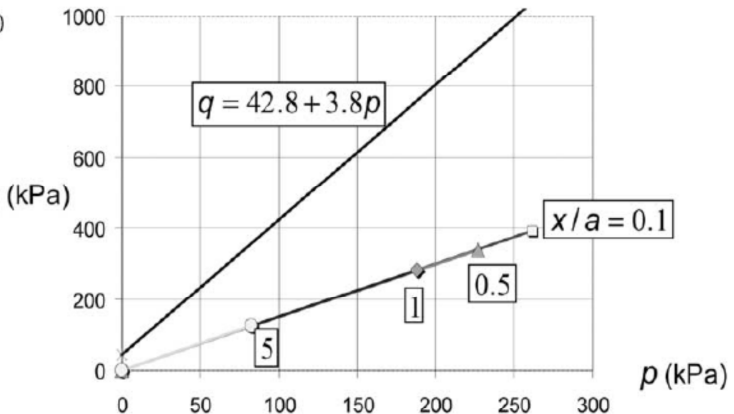

Fig. 11. (a) Residual stress profiles for increasing values of $N$; (b) residual stress paths in the (p,q)-plane

coefficients $v^{*}$ and $v$ have been varied independently, in order to assess their respective influence on the simulation results. The value of the Poisson's ratio $v$ being first kept constant equal to 0.3 , simulations have been carried out for different values of $v^{*}$ which governs the amplitude of lateral permanent strain as a function of axial permanent strain. Those values are ranging between 0.2 and 0.7 , which appear to be representative of such a parameter as deduced from preliminary experimental observations. Fig. 13(a) represents a series of curves showing the influence of $v^{*}$ on the accumulation of lateral permanent strains at a particular depth $x / a=1$, whereas Fig. 13(b) exhibits the influence of the same parameter on the global residual settlement predictions.

As it is quite apparent from the latter figure, the rate of accumulation of residual settlement is a decreasing function of $v^{*}$, so that by way of example, the global normalized residual settlement resulting from the application of one million load cycles, may vary from 


\section{$\delta^{r}(N) / a$}

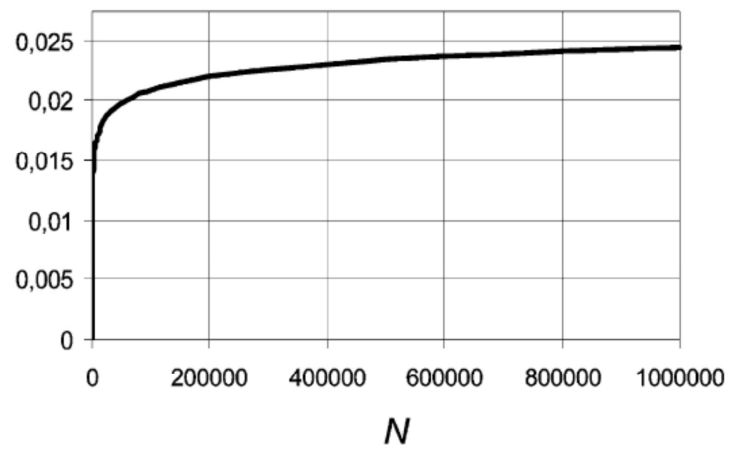

Fig. 12. Platform residual settlement as a function of repeated traffic loading.

$2 \times 10^{-2}$ for $v^{*}=0.7$ to $4 \times 10^{-2}$ for $v^{*}=0.2$, thus showing the decisive influence of this parameter.

A possible explanation of such a result may be found in Eq. (31), where it is to be noted that the axial permanent strain $\varepsilon_{1}^{p}$ is negative (contraction), while the lateral component $\varepsilon_{2}^{p}$ is all the more positive (extension) as the value of $v^{*}$ is important, with a correlative reduction of the residual settlement. More precisely, the residual surface settlement may be rewritten from Eqs. (31) and (19) as:

$\delta^{r}(N)=\left(1-2 v^{*} \frac{v}{1-v}\right)\left[-\int_{0}^{H} \varepsilon_{1}^{p}(x, N) \mathrm{d} x\right]$

Assuming that $v^{*}$ has a limited influence on the value of the integral of the vertical permanent strain, so that the term placed into brackets may be considered as an approximately constant positive quantity, Eq. (36) clearly demonstrates that the total residual settlement is a linearly decreasing function of $v^{*}$.

The same kind of observation can be made as regards the influence of the Poisson ratio $v$ on the results of the simulation. Curves giving the evolution of axial and lateral permanent strains at a particular depth level have been drawn in Fig. 14 for different values of this ratio. It appears that the amplitude of those strains reach their maximum values for $v=0$, with a significant decrease as it tends to 0.5 . This is mainly to be attributed to the fact that the reference stress cycles, and hence the rate of production of permanent strains, are dependent on the Poisson ratio, as previously shown by Fig. 6 .

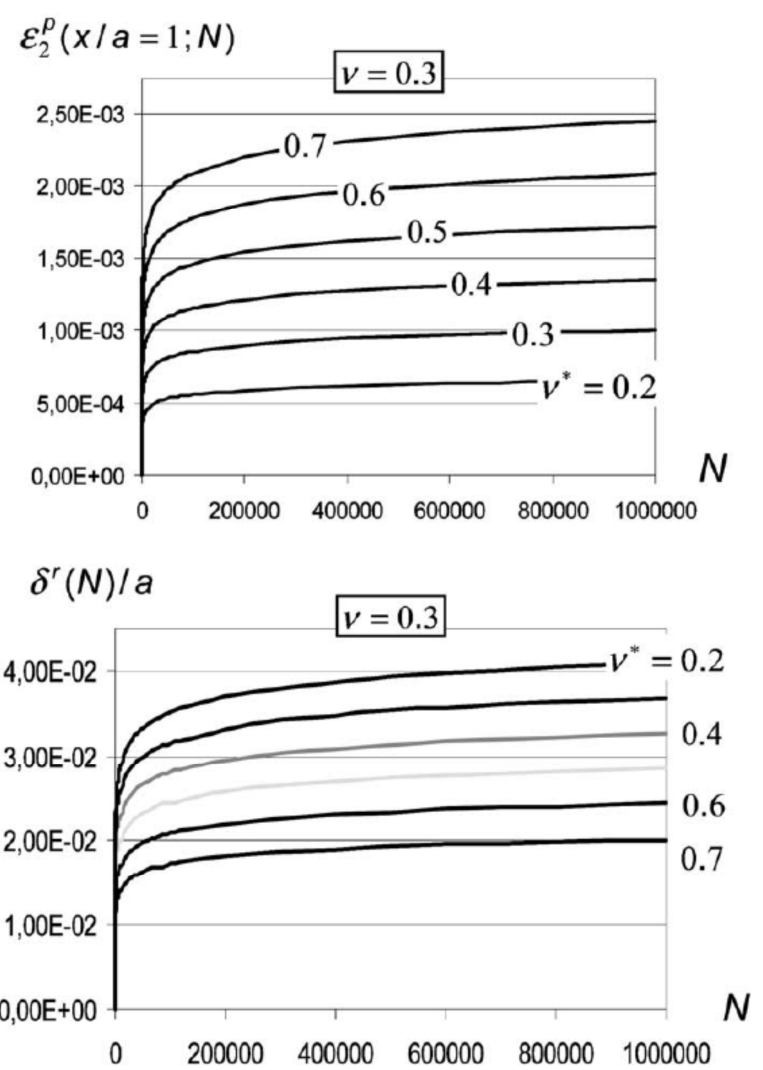

Fig. 13. Influence of $v^{*}$ on the evolutions of the lateral permanent strain and residual settlement as a function of $N$. 


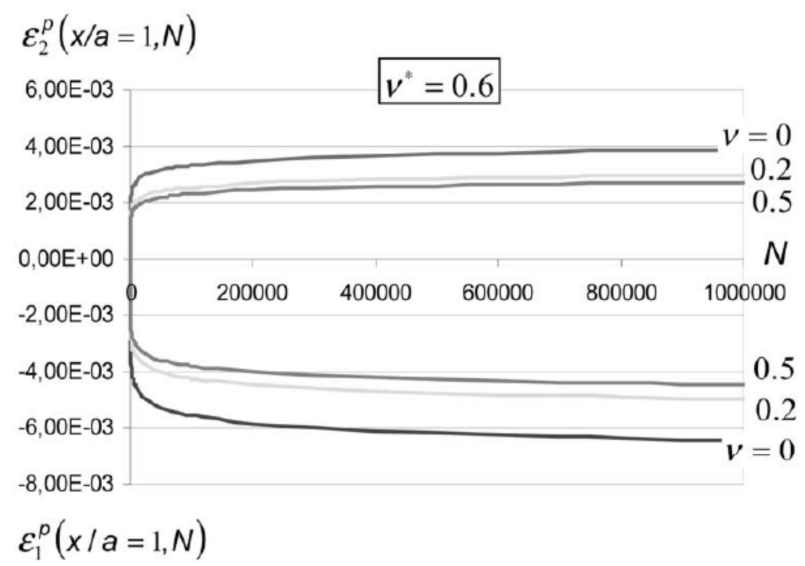

Fig. 14. Curves giving the variations of axial and lateral permanent strains for different values of $v$

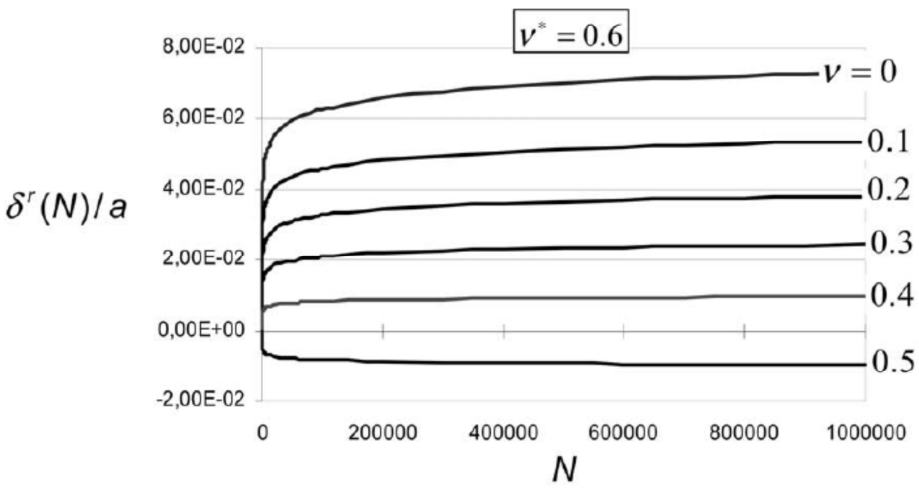

Fig. 15. Influence of the Poisson ratio on the evolution of residual surface settlement.

The influence of the value adopted for the material Poisson ratio on the residual surface settlement is still more pronounced, as shown by Fig. 15. It even appears that for a value comprised between 0.4 and 0.5 , the sign of the settlement is reversed, which means that for values of $v$ close to 0.5 (material elastic incompressibility), the residual surface displacement of the platform corresponds to an upheaval phenomenon. Again, this apparently surprising result could be explained from examining Eq. (35). Indeed, since the integral term put into brackets always remains positive, the sign of the surface settlement is given by that of the expression placed before, which only depends on $v$ and $v^{*}$. It follows that the condition for which the sign of the residual surface displacement is becoming negative (ground upheaval), writes

$$
v \geqslant \frac{1}{1+2 v^{*}}
$$

Since the Poisson ratio cannot exceed the value 0.5 , it appears that such a situation may only occur for $v^{*} \geqslant 1 / 2$, that is for a material exhibiting permanent strain volume dilatancy.

\section{Concluding remarks and perspectives}

The feasibility of the computational approach proposed for predicting the residual settlement of a pavement or railway platform under traffic loading, has been demonstrated on a simplified illustrative model. The following conclusions and perspectives as regards the future research trends in this field, could be drawn from this preliminary study.

(a) It should first be reminded that one of the essential features upon which the proposed method is based, lies in the possibility of formulating a cyclic constitutive law for the different 
granular materials making up the traffic platform. Such a constitutive law is primarily aimed at describing the permanent strains experienced by the materials subject to various cyclic solicitations. Although a quite significant effort has already been devoted to this question, it appears that an extensive experimental programme is still necessary in the forthcoming years for achieving such a reliable formulation within a consistent mechanical framework.

(b) In this context, two points deserve more particular attention. Referring to repeated triaxial loading tests, there is an urgent need to specify the respective evolutions of both the axial and lateral permanent strain components, which has been temporarily taken into account through the introduction of a constant ratio $v^{*}$. Furthermore, there remains to be seen how it is possible to generalize the constitutive formulation to stress cycles with rotating principal directions from triaxial compression tests where the principal stress directions remain fixed. In short, one is looking for establishing a formulation which is somewhat reminiscent of the classical "flow rule" introduced for modelling plastic deformations.

(c) The procedure applied here to a simplified model of traffic platform is meant to be implemented in the case of more realistic configurations, namely for the design and long term settlement prediction of transportation infrastructures such as a road pavement or a railway platform. This will obviously require the use of more sophisticated numerical tools, such as finite element or boundary element codes, to be incorporated in the general numerical scheme sketched in Fig. 4, which has been set up in this contribution. The adaptation of an existing software package (finite element computer code CESAR), aimed at performing the different elastic calculations involved at each step of the numerical procedure, is currently under way.

(d) Finally, among the main assumptions made in this paper, is the fact that the constituent materials behave as linear elastic media, resulting in decisive simplifications, such as the possibility of performing one single calculation for computing the reference stress cycles induced by the traffic loading at each point of the structure, independently from its residual state. Actually, it appears that materials such as unbound granular aggregates exhibit a reversible, but highly non linear behaviour, which can be captured through models such as that proposed by Boyce [18] on the basis of experimental data. One of the major improvement of the settlement prediction method proposed here, which could be expected in the near future, would therefore consist in integrating this kind of non linear constitutive law in the different elastic calculations.

\section{References}

[1] Guérin N. Experimental and numerical approach to the behaviour of railway ballast [in French]. PhD thesis, ENPC, Paris; 1996.

[2] Bodin V. Analysis of the global response of a ballast railway track under vertical and lateral loading [in French]. $\mathrm{PhD}$ thesis, ENPC, Paris; 2001

[3] Ponter ARS. The analysis of cyclically loaded creeping structure fort short cycle times. Int J Solids Structures 1976;12:809-25.

[4] Shahrour I, Meimon Y. Application of the homogenization method to the calculation of marine foundations subjected to periodic loads. NUMOG IV, Swansea 1992:601-8.

[5] Shahrour I, Meimon Y. Calculation of marine foundations subjected to repeated loads by means of the homogenization method. Computers and Geotechnics 1995;17:93-106.

[6] Monosmith CL, Ogawa N, Freeme CR. Permanent deformation characteristics of subsoil due to repeated loading. Trans Res Rec 1975;537:1-17

[7] Li D, Selig ET. Cumulative plastic deformation for fine-grained subgrade soils. J Geotech Eng 1996;122(12):1006-13.

[8] Chai J-C, Miura N. Traffic-load-induced permanent deformation of road on soft subsoil. J Geotech Eng 2002;128(11):90716.

[9] Li D, Selig ET. Method for railway track foundation design. II application. J Geotech Eng 1998;124(4):323-9.

[10] Raymond GP, Williams DR. Repeated Load Triaxial Tests on a Dolomite Ballast. J Geotech Eng 1978;104:1013-29.

[11] Alva-Hurtado, JED. A methodology to predict the elastic and inelastic behavior of railroad ballast. $\mathrm{PhD}$ thesis, University of Massachusetts; 1980.

[12] Hornych P, Corte JF, Paute JL. Etude des déformations permanentes sous chargement répétés de trois graves non traitées. Bull liaison P et Ch 1993; 184:45-55.

[13] Lekarp F, Dawson A. Modelling permanent deformation behaviour of unbound granular materials. Construction and Building Materials 1998;12(1):9-18.

[14] Gidel G, Hornych P, Chauvin JJ, Breysse D, Denis D. Nouvelle approche pour l'étude des déformations permanentes des graves non traitées à l'appareil triaxial à chargements répétés. Bull liaison P et Ch 2001;233:5-21.

[15] Dormieux L, Auriault JL, Coussy O. Pore pressures generation in a seabed subjected to wave loading. Eur J Mech, A/Solids 1993; 12(6):773-801.

[16] Pecker A, Prevost JH, Dormieux L. Analysis of pore pressure generation and dissipation in cohesionless materials during seismic loading. J Earthquake Eng 2001;5(4):441-64.

[17] Poulos HG, Davis EHElastic solutions for soil and rock mechanics. Centre for geotechnical research. University of Sydney; 1991.

[18] Boyce HR. A non linear model for the elastic behaviour of granular materials under repeated loading. Int Symp on Soils under Cyclic and Transient Loading, Swansea 1980;1:285-94. 